Gynaecological Cancer

\title{
CyberKnife Stereotactic Ablative Radiotherapy for Recurrent or Oligometastatic Gynecological Cancers
}

\author{
Tejinder Kataria ${ }^{1}$ Pushpa Naga ${ }^{1}$ Susovan Banerjee ${ }^{1,0}$ \\ Manoj Tayal ${ }^{1}$ Shyam Singh Bisht ${ }^{1}$ \\ ${ }^{1}$ Division of Radiation Oncology, Medanta - The Medicity, Gurgaon, \\ Haryana, India
}

South Asian J Cancer 2021;10:107-111.
Address for correspondence Susovan Banerjee, MD, Division of Radiation Oncology, Medanta - The Medicity, Sector 38, Gurgaon 122001, Haryana, India (e-mail: drsusovan@gmail.com).

\author{
Abstract

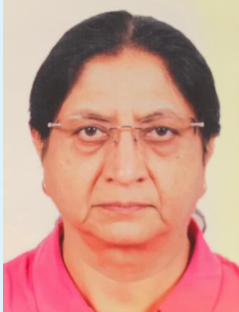 \\ T. Kataria \\ Keywords \\ - gynecological cancer \\ - recurrent \\ - oligometastasis \\ - stereotactic body \\ radiation therapy \\ - CyberKnife \\ - survivals
}

\section{Deepak Gupta $\quad$ Kushal Narang ${ }^{1}$}


or metastatic tumors in the recent years. ${ }^{4-7}$ Use of CyberKnife SABR as a salvage treatment option in unresectable, limited recurrent/metastatic gynecologic malignancies, such as cervical, vaginal, vulvar, and uterine cancer, is a relatively new concept. ${ }^{8,9}$ Its safety and efficacy in this setting is not been reported.

With an aim to evaluate and report our clinical experience of CyberKnife SABR in recurrent or oligometastatic gynecologic cancers, the electronic health information center records of 20 cases treated consecutively from January 2013 to December 2019 were analyzed.

\section{Materials and Methods}

Between January 2013 and October 2019, patients with histologically proven recurrent or metastatic gynecological cancers who were treated with stereotactic radiotherapy using the CyberKnife VSI system (Accuray Inc., Sunnyvale, California, United States) were enrolled for the study. The electronic medical records of all patients were reviewed. Patient demographics including age, stage, tumor characteristics, treatment details, toxicity, and clinical outcome parameters were recorded, compiled, and analyzed.

\section{CyberKnife Stereotactic Ablative Radiotherapy}

CyberKnife irradiation was considered in case of unresectable, limited recurrent, $\leq 5$ metastatic lesions with controlled primary gynecologic disease and for residual disease in case of recurrent/metastatic lesions after surgery or systemic chemotherapy.

All the patients were evaluated with whole body positron emission tomography and computed tomography (PET/CT) scans prior to treatment. Written informed consent was obtained from each patient. Patients were immobilized in the required treatment positions with appropriate immobilization devices, either using thermoplastic masks or Vac-Lok Cushion (CIVCO Medical Solutions, Orange City, Iowa, United States).

CyberKnife planning CT simulation (Siemens Medical Systems, USA, Inc) was performed by acquisition of both noncontrast and contrast (intravenous, Iobitridol [Xenetix]) CT images with $1 \mathrm{~mm}$ slice thickness. Following acquisition, all image sets were transferred to Multiplan v4.6.0 (Accuray Inc.) treatment planning system. Tumor volumes and organ-at-risks (OARs) were contoured on noncontrast CT images according to the institutional protocol. The gross tumor volume (GTV) was defined as visible tumor on contrast planning enhanced CT, PET/CT, and/or magnetic resonance imaging (MRI) images fused for better target delineation. The GTV was considered to be identical as clinical target volume (CTV). The planning target volume (PTV) included a margin expansion of 2 to $5 \mathrm{~mm}$ to CTV.

Treatment planning was performed on noncontrast CT image data sets. The prescription doses were determined considering multiple factors, such as tumor volume, previous radiation doses, critical consideration of adjacent organs, and number of fractions. Dose was typically prescribed to the 70 to $85 \%$ isodose line of the maximum dose to cover 98 to
99\% of PTV (-Fig. 1). Plans were evaluated qualitatively by examining prescribed reference isodose to adequately cover the target volume in all dimensions and quantitatively by recording Dmean, D2\%, D95\%, and D98\% (the dose received by mean, $2 \%, 95 \%$, and $98 \%$, respectively) of the target volume, homogeneity indices (HIs), conformity indices (CIs), and dose to corresponding adjacent OAR. Dose fractionations were normalized to 2-Gy equivalent doses (Eq. D2) and biologically effective dose (BED) with $\alpha / \beta$ ratio of $10 \mathrm{~Gy}$ for tumor and $3 \mathrm{~Gy}$ for normal tissues. Treatment was delivered on CyberKnife VSI system. Tumors tracking systems were used as indicated. Adequate bowel preparation instructions were given at simulation and treatment. All patients were advised low fiber diet, laxatives as required, and activated charcoal tablets to minimize the uncertainties related to bowel movement and bowel gas. Additional adjuvant treatments including systemic chemotherapy, hormone therapy, and targeted therapy were considered whenever indicated.

PET-based radiological imaging and clinical follow-up were performed at 10 to 12 weeks posttreatment for treatment response evaluation. Thereafter, all patients were followed-up with clinical examination every 3 months in first 2 years and PET/CT every year. Subsequent clinical examination was scheduled at 6 months for 3 more years.

Tumor response was assessed using Response Evaluation and Criteria in Solid Tumors. Local failure was defined as an increase in the targeted tumor size or the presence of a new lesion in the radiation field. However, if a new lesion developed outside the radiation field, it was interpreted as distant metastasis.

Acute and late toxicities were defined as symptoms that developed within 3 months posttreatment or later, respectively, and graded according to the Radiation Therapy Oncology Group/European Organization for Research and the Treatment of Cancer (RTOG/EORTC) radiation toxicity scoring system.

\section{Statistical Analyses}

Statistical analysis was performed using Statistical Program for Social Sciences software for Windows (SPSS Inc., Version 26, Chicago, Illinois, United States). Kaplan-Meier's method was used to calculate local control (LC) rates.

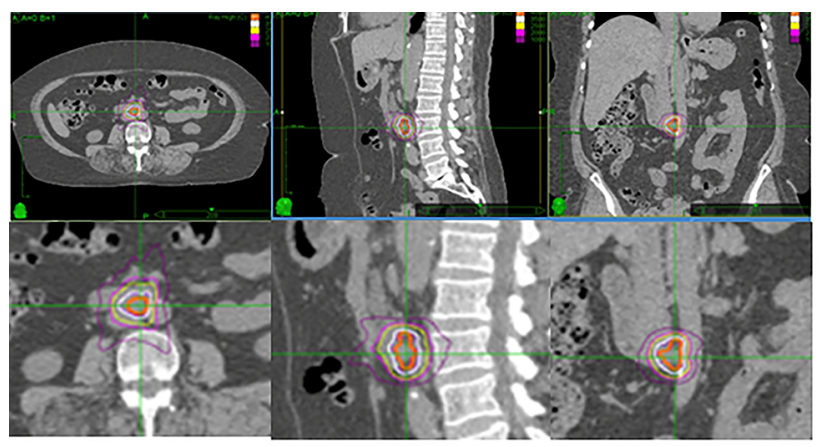

Fig. 1 CyberKnife planning three-dimensional (3D) computed tomography images showing target volumes and isodose distribution in different views. 


\section{Results}

\section{Patient and Tumor Characteristics}

- Table 1shows patient and tumor characteristics in detail. Twenty primary gynecological cancer patients with 25 biopsy-proven recurrent or oligometastatic lesions underwent CyberKnife SABR. With a median age of 60 years (range, 40-82; mean, 59 years), 8 patients (40\%) were primary cervical, 6 (30\%) were ovarian, 5 (25\%) were endometrial, and 1 patient (5\%) had vulvar cancer. Of these, 17 patients were proven to have isolated recurrent/metastatic disease and 3 had more than one recurrent/metastatic disease ( 2 patients had two lesions and 1 patient had four lesions) seen on PET/CT. Four (16\%) intracranial and 21 (84\%) extracranial lesions including 14 (67\%) extrapelvic and 7 (33\%) pelvic lesions were treated. Fourteen (70\%) patients had prior pelvic radiation therapy (RT) as part of the initial primary treatment and 5 (25\%) of 25 targets were within previously irradiated areas, 2 (10\%) lesions were partially overlapped with previous RT field and the remaining 7 had lesions outside RT field. Median time to recurrence among patients with prior RT was 32 months (range, 5-120 months).

Table 1 Patient and tumor characteristics (20 patients and 25 target lesions)

\begin{tabular}{|c|c|c|}
\hline Parameters & Classification & $N(\%)$ \\
\hline Age, y $(n=20)$ & Median (range) & $60(40-82)$ \\
\hline \multirow[t]{4}{*}{ Primary tumor $(n=20)$} & Cervix & $8(40)$ \\
\hline & Endometrium & $5(25)$ \\
\hline & Ovary & $6(30)$ \\
\hline & Vulva & $1(5)$ \\
\hline \multirow[t]{3}{*}{ Histology $(n=20)$} & $\begin{array}{l}\text { Squamous cell } \\
\text { carcinoma }\end{array}$ & $7(35)$ \\
\hline & Adenocarcinoma & $12(60)$ \\
\hline & Others & $1(5)$ \\
\hline \multirow{2}{*}{$\begin{array}{l}\text { Previous history of } \\
\text { pelvic radiation }(n=20)\end{array}$} & Yes & $14(70)$ \\
\hline & No & $6(30)$ \\
\hline \multirow[t]{2}{*}{$\begin{array}{l}\text { CK-SABR treatment } \\
\text { setting }(n=25)\end{array}$} & $\begin{array}{l}\text { 1st time recurrent/ } \\
\text { metastatic }\end{array}$ & $13(52)$ \\
\hline & $\begin{array}{l}\text { Re-recurrent/ } \\
\text { metastatic }\end{array}$ & $12(48)$ \\
\hline \multirow[t]{7}{*}{ CK-SABR site $(n=25)$} & $\begin{array}{l}\text { Lymph nodes } \\
\text { - Paraaortic }\end{array}$ & $9(36)$ \\
\hline & $\begin{array}{l}\text { Lymph nodes } \\
\text { - Others }\end{array}$ & $3(12)$ \\
\hline & Lung & $5(20)$ \\
\hline & Brain & $4(16)$ \\
\hline & Bone & $2(8)$ \\
\hline & Soft tissue deposit & $1(4)$ \\
\hline & Vaginal vault & $1(4)$ \\
\hline
\end{tabular}

Abbreviations: CK, CyberKnife; SABR, stereotactic ablative radiotherapy.

\section{Treatment Characteristics}

CyberKnife SABR was performed as a first-line therapy in 11 (55\%) patients and for recurrent/metastatic disease in 9 (45\%) patients. A total of 25 treated lesions included 13 (52\%) postchemotherapy residual recurrent/metastatic disease, 5 (20\%) new metastatic lesions, 3 (15\%) post-RT rerecurrent lesions, 2 (8\%) postop post-RT post-chemo residual, 1 (4\%) post-chemo post-RT residual, and 1 (4\%) postop residual recurrent/metastatic disease.

The target volumes, GTV ranged from 0.7 to $30 \mathrm{~cm}^{3}$ (mean $9 \mathrm{~cm}^{3}$, median $8 \mathrm{~cm}^{3}$ ) and the PTV ranged from 3 to $280 \mathrm{~cm}^{3}$ (mean $40 \mathrm{~cm}^{3}$, median $17 \mathrm{~cm}^{3}$ ). Dose was prescribed to the 60 to $86 \%$ (median $80 \%$ ) isodose line. The prescription dose ranged from 15 to $35 \mathrm{~Gy}$ (median $30 \mathrm{~Gy}$ ) and the median SABR dose delivered was 60 Gy BED (range, 42-133) in an average of four fractions (range, 1-6). In two patients with solitary brain metastasis, single-fraction stereotactic radio surgery was done delivering 20 Gy to PTV. Overall, mean ( \pm standard deviation [SD]) CyberKnife SABR duration was 6 days ( \pm 3 days). Mean $( \pm$ SD) percentage dose received by Dmean, D2\%, D95\%, and D98\% was 110\% ( \pm 6.1$), 123 \%$ ( \pm 6.1 ), $99.5 \%( \pm 8.5)$, and $96.4 \%( \pm 9.8)$, respectively. The mean value of HI was 27 (range, 11-56) and CI was 1.2 (range, 1-2). All targets were tracked during treatment delivery using Xsight Spine ( $n=14,56 \%), 6 \mathrm{D}$ skull $(n=4,16 \%)$, fiducials ( $n=4,16 \%)$, and synchrony $(n=3,12 \%)$ tracking systems. After completion of CyberKnife SABR, 9 patients continued to receive additional treatment including systemic chemotherapy in 6 and hormone therapy in 3 patients.

\section{Treatment Response and Follow-Up}

Eighteen patients underwent PET/CT and one had MRI scan at approximately 8 to 12 weeks after treatment. One patient was lost to follow-up after completing SABR, and hence response could not be evaluated. On response evaluation imaging by first 3 months, 9 (47.5\%), 5 (26\%), 4 (21\%), and 1 (5.5\%) of the 19 patients had complete response (CR), partial response $(\mathrm{PR})$, progressive disease, and stable disease, respectively. During the subsequent follow-up imaging, two patients of PR achieved CR and six more patients ( 3 of 9 CR patients and 3 of 5 PR) were diagnosed with disease relapse/progression.

Therefore, out of 19 patients, 8 (42\%) patients were disease-free and remaining 11 (58\%) were detected with disease progression, including local progression and/or distant metastasis. Of these, two patients had local progression, eight had distant visceral/lymph nodal metastasis, and one had both local progression and distant metastasis. Among 11 relapses, a patient with local progression was salvaged with surgery whereas remaining patients received either palliative chemotherapy or radiation or symptomatic treatment only.

With a mean follow-up of 18 (range, 2-70) months, all of the 7 disease-free patients along with one patient with second primary meningioma were alive and healthy with average follow-up period of 25 months. Of the 11 relapses, one of the relapsed patients died due to the disease after 7 months of treatment completion, and 3 patients were lost to follow-up after a minimum follow-up period of 4 months. 


\section{Local Control and Toxicity}

Local tumor control was achieved in $82 \%$ of patients. Overall acute toxicity was observed in 9 (45\%) of the 20 patients. The most common grade 1 to 2 adverse events during the treatment were nausea and vomiting ( $n=3,15 \%)$, gastrointestinal (GI) toxicity $(n=2,10 \%)$, fatigue $(n=2,10 \%)$, and genitourinary toxicity $(n=1,5 \%)$. In one patient with grade $2 \mathrm{GI}$ toxicity, planned treatment was concluded a fraction earlier in view of poor tolerance and worsening general condition and was lost to response evaluation later. No acute grade 3 to 4 toxicities occurred, and no late toxicities were recorded.

\section{Discussion}

The management of patients with recurrent or oligometastatic gynecological cancers is challenging. Achieving an early and optimal LC of recurrent or oligometastatic lesion in cases with controlled primary is the key factor for favorable outcome. Therapeutic efficacies of various local treatment modalities including RT, surgery, radiofrequency ablative therapy, and combination therapy have been explored. Considering its efficacy and minimum invasiveness, RT is a more viable and feasible option in most cases in contrast to surgery. However, conventional radiation technique is limited by the virtue of its potential toxicity to OARs to deliver radical doses. SABR using CyberKnife enables to deliver high radiation dose with submillimeter precision to the target allowing narrow margins and thus relatively sparing nearby OAR structures. ${ }^{10-12}$

Our series consisted of $4(16 \%)$ intracranial, 14 (56\%) extrapelvic, and 7 (28\%) intrapelvic lesions. The observed 1-year overall survival (OS) was $94 \%$ and local tumor control was achieved in $82 \%$ of patients.

An intriguing observation of our study is that more than half of the cases were extrapelvic lesions in contrary to the published series. ${ }^{13}$ Patients with extrapelvic recurrent/metastatic disease generally have poor clinical outcomes, with a reported 5-year OS of less than $20 \%{ }^{14}$ It is noteworthy that, in our series 11 of the 14 patients (78\%) with extrapelvic lesions had achieved LC by 1 year with median OS of 19 months. Notably, patients with solitary recurrences had a relatively better progression-free survival (PFS) and OS than those with multiple lesions (2-year PFS, $56 \%$ vs. $40 \%$ and 2 -year OS, $96 \%$ vs. $88 \%$ ).

There is no much literature published or any standard recommendations for dose fractionation schedule for the use of SABR for recurrent or oligometastatic gynecological cancers. With the available evidence, the BED doses delivered vary from 60 to $90 \mathrm{~Gy}$. In our series, patients received a median SABR dose of 60 Gy BED (range, 42-133) in an average of four fractions (range, 1-6), which is slightly lower in comparison to another series reported by a Korean group. ${ }^{14}$ Because of the diverse recurrent/metastatic sites and heterogeneous patient status, it is difficult to determine the optimal dose for tumor control. It might be reasonable to determine the treatment dose individually based on dose constraints of adjacent organs.
Recently, owing to availability of more sensitive methods of detection, recurrent/oligometastatic states are more frequently identified than before. Site of recurrence/metastasis, type of initial primary therapy, and disease-free interval have vital implication on further management of these lesions. ${ }^{14}$ In general, an early identification and LC of recurrences before progression to disseminated disease may improve patient outcomes.

The limitations of our study include small sample size, retrospective nature of data, and heterogeneous target population. Nevertheless, it is an early experience of a single institution representing data from a low-middle income country in a realistic scenario. Our data indicate that patients with recurrent or oligometastatic gynecological cancers do not invariably have a dismal prognosis. CyberKnife SABR can be an effective local therapy for recurrent/metastatic gynecological cancers with curative potential. It would be interesting to explore outcome of SABR with further addition of systemic therapy intervention strategies like immunotherapy and/or targeted therapy for better salvage rates.

\section{Conclusion}

CyberKnife SABR offers an effective and safe approach for selected cases of recurrent or oligometastatic gynecologic cancers. Initial outcomes are encouraging with good LC and acceptable toxicity. The efficacy and toxicity need to be evaluated over the long term. Further, large-scale studies are required to define optimal target doses, dose-response relationship, and OAR limits.

\author{
Funding \\ None. \\ Conflict of Interest \\ None declared.
}

\section{References}

1 Brady LW, Perez CA, Bedwinek JM. Failure patterns in gynecologic cancer. Int J Radiat Oncol Biol Phys 1986;12(4):549-557

2 Niibe Y, Hayakawa K. Oligometastases and oligo-recurrence: the new era of cancer therapy. Jpn J Clin Oncol 2010;40(2):107-111

3 Choi CW, Cho CK, Yoo SY, et al. Image-guided stereotactic body radiation therapy in patients with isolated para-aortic lymph node metastases from uterine cervical and corpus cancer. Int J Radiat Oncol Biol Phys 2009;74(1):147-153

4 Pan H, Simpson DR, Mell LK, Mundt AJ, Lawson JD. A survey of stereotactic body radiotherapy use in the United States. Cancer 2011;117(19):4566-4572

5 Correa RJ, Salama JK, Milano MT, Palma DA. Stereotactic body radiotherapy for oligometastasis: Opportunities for biology to guide clinical management. Cancer J 2016;22(4):247-256

6 Lewis SL, Porceddu S, Nakamura N, et al. Definitive stereotactic body radiotherapy (SBRT) for extracranial oligometastases: an international survey of $>1000$ radiation oncologists. Am J Clin Oncol 2017;40(4):418-422

7 Gomez DR, Blumenschein GR Jr, Lee JJ, et al. Local consolidative therapy versus maintenance therapy or observation for patients with oligometastatic non-small-cell lung cancer without progression after first-line systemic therapy: a multicentre, randomised, controlled, phase 2 study. Lancet Oncol 2016;17(12):1672-1682 
8 Laliscia C, Fabrini MG, Delishaj D, et al. Clinical outcomes of stereotactic body radiotherapy in oligometastatic gynecological cancer. Int J Gynecol Cancer 2017;27(2):396-402

9 Kunos C, Chen W, DeBernardo R, et al. Stereotactic body radiosurgery for pelvic relapse of gynecologic malignancies. Technol Cancer Res Treat 2009;8(5):393-400

10 Mesko S, Sandler K, Cohen J, Konecny G, Steinberg M, Kamrava M. Clinical outcomes for stereotactic ablative radiotherapy in oligometastatic and oligoprogressive gynecological malignancies. Int J Gynecol Cancer 2017;27(2):403-408

11 Mendez LC, Leung E, Cheung P, Barbera L. The role of stereotactic ablative body radiotherapy in gynaecological cancers: a systematic review. Clin Oncol (R Coll Radiol) 2017;29(6):378-384
12 Palma DA, Olson R, Harrow S, et al. Stereotactic ablative radiotherapy for the comprehensive treatment of oligometastatic cancers: long-term results of the SABR-COMET phase II randomized trial. J Clin Oncol 2020;38(25):2830-2838

13 Hasan S, Ricco A, Jenkins K, et al. Survival and control prognosticators of recurrent gynecological malignancies of the pelvis and para-aortic region treated with stereotactic body radiation therapy. Front Oncol 2016;6:249

14 Park HJ, Chang AR, Seo Y, et al. Stereotactic body radiotherapy for recurrent or oligometastatic uterine cervix cancer: a cooperative study of the Korean Radiation Oncology Group (KROG 14-11) Anticancer Res 2015;35(9):5103-5110Photo To Come 